\title{
Does prospective payment increase hospital (in)efficiency? Evidence from the Swiss hospital sector
}

\author{
Philippe K. Widmer
}

Received: 4 July 2013/ Accepted: 14 March 2014/Published online: 9 April 2014

(C) Springer-Verlag Berlin Heidelberg 2014

\begin{abstract}
Several European countries have followed the USA in introducing prospective payment for hospitals with the expectation of achieving cost efficiency gains. This article examines whether theoretical expectations of cost efficiency gains can be empirically confirmed. In contrast to previous studies, the analysis of hospitals in Switzerland provides a comparison of a retrospective per diem payment system with a prospective global budget and a payment per patient case system. Using a sample of approximately 90 public financed Swiss hospitals during the years 2004-2009 and Bayesian inference of a standard and a random parameter frontier model, cost efficiency gains are found, particularly with payment per patient case. Prospective payment, designed to put hospitals at operating risk, is more effective in terms of cost reduction than the retrospective alternative. However, hospitals are heterogeneous with respect to their production technologies, making a random parameter frontier model the superior specification for Switzerland.
\end{abstract}

Keywords Hospital inefficiency - Prospective payment system - Bayesian inference $\cdot$ Stochastic frontier analysis

JEL Classification $\quad \mathrm{C} 11 \cdot \mathrm{C} 23 \cdot \mathrm{D} 24 \cdot \mathrm{I} 18$

The author would like to thank Peter Zweifel for his valuable support.

P. K. Widmer $(\bowtie)$

Department of Economics, University of Zurich,

Hottingerstrasse 10, 8032 Zurich, Switzerland

e-mail: philippe.widmer@econ.uzh.ch

P. K. Widmer

Polynomics Inc., Olten, Switzerland

\section{Introduction}

Growth of health care expenditure over the last several decades has highlighted the need for health care reforms in order to contain future cost increases. One promising approach, which was first implemented in the USA and was recently adapted by many European countries, involves the transition from retrospective (RPS) to prospective (PPS) hospital payment systems (for an overview of reforms in Europe, see [30, 32]). The assumption is that a change to fixed, predetermined payment would place hospitals at operating risk, making them increase their cost efficiency.

Even though there are convincing theoretical arguments for cost reductions and efficiency gains $[3,7,26]$, empirical evidence is lacking. The link between efficiency gains and PPS has yet to be demonstrated for the US Medicare reform of 1983, which switched from RPS to payment per patient case, or for any of the European countries that moved from RPS to payment per patient case or a global budget (see Sect. 2 for further information on payment systems). For example, Borden [6] found no significant efficiency gains for 93 New Jersey hospitals between 1979 and 1984. Similar results were obtained by Chern and Wan [9] when they examined the catch-up effect of technically inefficient hospitals in Virginia from 1984 to 1993. Inefficient hospitals became even more inefficient in 1993, contrary to what would be expected of PPS. However, efficiency gains were shown by Morey and Dittman [25], who analyzed the technical inefficiency of 105 hospitals in North Carolina. The results of European reforms remain inconclusive, too [29, 36]. Whereas no efficiency gains were found in Austrian hospitals after funding shifted from per diem payment to global budgets in 1997 [33] and in Germany after the switch to per case payment in 2004 [17], gains were found in Portugal [11], Finland [22], and 
Norway [4]. Since PPS is well known to cause higher costs of administration and monitoring costs, which are abstracted from theoretical models and neglected in most empirical research, its overall cost-reducing effect could be overstated.

However, these inconclusive results reported are likely due to a lack of analytical rigor. In particular, although it is widely accepted that hospitals are rather heterogeneous in their production of health care services [38], previous applications of data envelopment analysis (DEA) and stochastic frontier analysis (SFA) have been based on the assumption of homogeneous technology. Furthermore, it is well known that the popular applied two-stage DEA approach yields biased estimates because it fails to account for the possible correlation of the independent variables with the inputs and outputs of the first-stage DEA [31]. Finally, as most countries switched to PPS at the country level only recently, the time series available for withintreatment analysis have been very short. Most studies analyze a time series of $4-5$ years, which may be too short to draw any reliable conclusions. Changes could be driven by unobserved exogenous shocks, such as new medical technologies or inflation, that occurred during implementation of PPS [22].

In order to overcome the limitations of previous studies, this article compares retrospective per diem payment with a prospective global budget as well as prospective payment per patient case using data from Switzerland. The Swiss experience is of interest because some member states (cantons) changed to different variants of PPS while others remained with RPS. The contribution of this article is threefold. First, it extends previous work by implementing a random parameter frontier model to control for the importance of unobserved heterogeneity among six hospital categories. Second, it examines the robustness of findings with respect to assumptions concerning production technology. Third, it determines whether theoretical expectations of cost savings can be confirmed by relating calculated inefficiency scores to the three payment systems. Estimates are based on the extended single-step approach by Battese and Coelli [2]. However, the observation period (2004-2009) is rather short again.

The empirical analysis reveals two key results. First, the random parameter frontier model, which accounts for unobserved heterogeneity, is more robust and has higher explanatory power than the standard cost frontier model. Therefore, correcting among hospitals for heterogeneity is crucial in deriving meaningful inefficiency scores. Second, PPS is negatively correlated with hospital cost inefficiency, particularly PPS of the per patient case type. Prospective payment, designed to expose hospitals to operating risk, is more effective than retrospective payment in containing hospital cost.

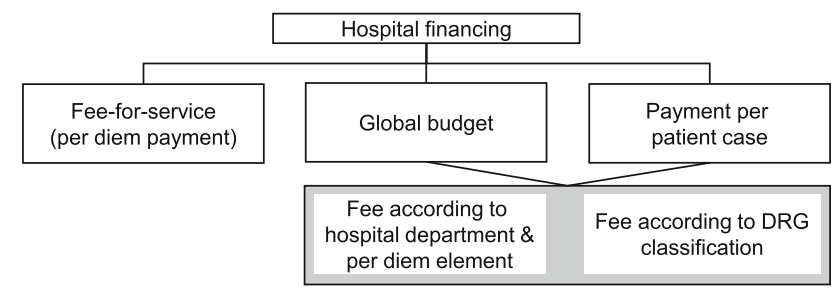

Fig. 1 Hospital payment systems in Switzerland. DRG Diagnostic Related Group

The remainder of this paper is structured as follows. Section 2 gives an overview of the different prospective and retrospective payment systems that coexisted in Switzerland between 2004 and 2009. Section 3 outlines the standard and random parameter frontier models, respectively. Section 4 describes the database as well as the econometric specifications. Finally, Sect. 5 presents the results of the cost frontier models and informs about the determinants of inefficiency.

\section{Introduction to Swiss hospital financing}

The Swiss health care system has been shaped by the country's decentralized federal structure, in which 26 cantons are responsible for providing health care services to their residents. The hospital sector is no exception, with capacity planning and quality monitoring falling in the domain of cantons. Hospitals that are qualified to provide health care to socially insured patients figure on a cantonal list. However, this does not imply that hospital financing only comes from cantonal sources. On the contrary, health insurers are obliged to cover up to $45 \%$ of operating costs, resulting in a dual system where cantons finance both the cost residual and capital investment. ${ }^{1}$

Increasing health care costs have induced many cantons to revise their system of hospital payment. The new federal law on social health insurance of 1994 (effective from 1996) gave them legal authority to control hospital operating costs. Since not all cantons exercised this authority, various RPS and PPS schemes coexisted in Switzerland (see Fig. 1 for an overview).

Prior to 1996, cantons primarily used retrospective costbased per diem or fee-for-service to pay hospitals for their services. Remuneration was equal to reported cost, making bankruptcy impossible. Rarely, a hospital was closed because cantonal authorities sought to reduce overcapacity.

\footnotetext{
${ }^{1}$ Health insurers cover more than $45 \%$ of operating costs in the case of privately owned hospitals which are not on the cantonal list. However, these are typically for-profit hospitals specializing in the treatment of patients having supplementary insurance coverage.
} 
Unsurprisingly, critics argued that there was little incentive for cost containment. Hence, after 1996 several cantons experimented with PPS in an attempt to create incentives for cost containment. The two alternatives included a global budget and payment per patient case (see Fig. 1). Under a global budget, hospitals of a given category are paid a fixed amount for a predetermined number of admissions regardless of the numbers of patients seeking care during the accounting period. Under payment per patient case, hospitals of a given category receive a fixed amount per admission regardless of the actual cost of treatment. In both cases, hospitals can retain a surplus but have to bear a loss, making them act to minimize cost.

However, incentives for cost minimization could be weakened in the Swiss case because many cantons still do not firmly exclude a bailout. This is especially true for those with a global budget system, allowing hospitals to renegotiate their budgets when confronted with unexpected cost increases. Hospitals thus continue to enjoy an implicit deficit guarantee which protects them against their operating risk and therefore weakens their incentive for cost minimization. Furthermore, the exact way payment per case is determined could also influence incentives. Two variants are widely used in Switzerland (see Fig. 1 again). The first variant fixes payment per admission as a department-specific average price, combined with a per diem element to control for differences in length of stay. The second variant uses a Swiss specification of the $\mathrm{DRG}^{2}$ classification system. In contrast to the first variant, payments are independent of length of stay, thus doing away with incentives to maximize length of stay and holding the promise of cost savings. While DRG systems contain provisions for the treatment of patients who are unusually expensive, these outlier payments apply to only a small portion of patients and are not directly related to length of stay.

An increasing number of cantons have changed to PPS. In 2004, only $38 \%$ of all Swiss hospitals were still reimbursed by per diem payments. Most hospitals had PPS, and almost $36 \%$ of them already used the DRG classification. In 2007, the number of hospitals under PPS increased even more, and most cantons used the DRG classification [24]. In 2007, Swiss parliament revised the insurance law to introduce a uniform DRG system in all cantons by 2012. Following the US Medicare reform of 1983 and the German reform of 2004, policy makers believe that the new reimbursement system would increase cost efficiency (see "Introduction"). This article aims to determine whether PPS (especially when combined with the DRG system is indeed more effective in

\footnotetext{
$\overline{2}$ Diagnostic Related Groups (DRGs) are a diagnosis classification system, designed to reflect resource requirements in hospital treatment. In Switzerland, AP-DRG, a non-profit organization, was created to define diagnostic groups and to establish their cost weights. In 2012, SwissDRG replaced the AP-DRG system.
}

containing hospital cost than the RPS alternative. The following two hypotheses are to be tested:

1. Hospitals under PPS are more cost-efficient than hospitals under RPS. Putting a hospital at operating risk should strengthen incentives for cost minimization (lower cost inefficiency).

2. Hospitals under PPS combined with the DRG classification are more cost-efficient than those under PPS combined with a per diem element. The fact that DRG systems do not account for longer length of stay should cause additional cost savings (lower cost inefficiency).

\section{Estimation models}

In order to analyze these hypotheses, hospital-specific inefficiency scores must first be derived from estimated cost frontiers. ${ }^{3}$ Two specifications serve to check for the importance of unobserved heterogeneity among hospitals. The first specification is the standard frontier model (SFM), which was first implemented by Aigner et al. [1] and Meeusen and van den Broeck [23]. It estimates inefficiency as the distance between a cost frontier and observed expenditure. Observable heterogeneity thereby is captured by shifting means of the inefficiency term (cf. [2, 18, 21]). The second specification is a random parameter frontier model (RPFM) that additionally controls for unobserved heterogeneity in technology parameters (for other applications, see [28, 37]). Inefficiency is estimated as a deviation from a cost frontier that differs according to type of hospital.

\section{Standard frontier model (SFM)}

The cost frontier for hospital $i=1, \ldots, N$ at time period $t=1, \ldots, T$ can be written as

$$
C_{i t}=C\left(Y_{i t}, W_{i t} ; \alpha, \beta\right)+\overbrace{u_{i t}+v_{i t}}^{\varepsilon_{i t}},
$$

with $C_{i t}$ representing operating expenditure, $Y_{i t}$ denoting the output vector, and $W_{i t}$ is the vector of input prices. $\alpha$ is the intercept and $\beta$ is a $(K \times 1)$ vector of unknown slope parameters. $C\left(Y_{i t}, W_{i t} ; \alpha, \beta\right)$ is the deterministic part of the cost frontier that remains to be specified for empirical estimation. Typically, this is either a Cobb-Douglas or a more flexible functional form (e.g., translog).

The error term $\varepsilon_{i t}$ is split into two additive components, random noise $v_{i t}$ and cost inefficiency $u_{i t}$. Random noise is normally distributed $v_{i t} \stackrel{i i d}{\sim} N\left[0, \sigma_{v}^{2}\right]$ with mean zero and variance $\sigma_{v}^{2}$. Hospital-specific inefficiency $u_{i t}$ is assumed to follow a one-sided distribution supported on the interval

\footnotetext{
${ }^{3}$ For an overview of inefficiency measurement methods, see [10, 20].
} 
$[0, \infty)$. The larger $u_{i t}$, the more cost inefficient a hospital and the greater the potential for cost savings.

Since the main purpose of this paper is to analyze the influence of PPS on efficiency, inefficiency is specified in accordance with Battese and Coelli [2] as a truncated normal distribution $u_{i t} \sim f_{N^{+}}\left[\bar{u}_{i t}, \sigma_{u}^{2}\right]$ with hospital-specific mean $\bar{u}_{i t}$ and variance $\sigma_{u}^{2}$. Mean inefficiency is hypothesized to be a linear function of $l=1, \ldots, L$ determinants $Z_{\text {lit }}$,

$\bar{u}_{i t}=\gamma_{o}+\sum_{l=1}^{L} \gamma_{l} Z_{l i t}+\varsigma_{i t}$,

where $\left[\gamma_{0}, \gamma_{l}\right]$ is an $(L+1)$ vector of unknown parameters to be estimated and $\varsigma_{i t}$ an unexplained source of hospitalspecific inefficiency.

\section{Random parameter frontier model (RPFM)}

One way to extend the SFM to account for unobserved heterogeneity is the random parameter frontier model, which derives inefficiency scores from hospital-specific cost frontiers. ${ }^{4}$ Here, $j=1, \ldots, 6$ exogenously given hospital categories are distinguished which are expected to reflect differences in production technology,

$$
\begin{aligned}
C_{i t} & =C\left(Y_{i t}, W_{i t} ; \alpha_{j}, \beta_{j}\right)+\overbrace{u_{i t}+v_{i t}}^{\varepsilon_{i t}}, \\
u_{i t} & \sim f_{N^{+}}\left[\bar{u}_{i t}, \sigma_{u}^{2}\right], \quad \bar{u}_{i t}=\gamma_{o}+\sum_{l=1}^{L} \gamma_{l} Z_{l i t}+\varsigma_{i t}, \\
\alpha_{j} & =\alpha+w_{j}, \\
\beta_{j} & =\beta+w_{j} .
\end{aligned}
$$

In contradistinction from the SFM, this specification allows inefficiency to be disentangled from unobservable heterogeneity through $\alpha_{j}=\alpha+w_{j}$ and slope parameters $\beta_{j}=$ $\beta+w_{j}$ that are specific to the six hospital categories (see Sect. 4.1). Time-invariant and category-specific heterogeneity is captured by $w_{j}$, which is a $[(K+1) \times 1]$ vector of random variables. Similar to Tsionas [37], $\alpha_{j}$ and $\beta_{j}$ are assumed to follow a multivariate normal distribution,

$$
\left(\begin{array}{c}
\alpha_{j} \\
\beta_{j}
\end{array}\right) \sim f_{M N}\left[\left(\begin{array}{c}
\bar{\alpha} \\
\bar{\beta}
\end{array}\right), \Sigma\right], \quad \text { with } \quad \Sigma \sim f_{W}\left[\begin{array}{cc}
\sigma_{\alpha}^{2} & \sigma_{\alpha, \beta} \\
\sigma_{\alpha, \beta} & \sigma_{\beta}^{2}
\end{array}\right],
$$

\footnotetext{
${ }^{4}$ It is worth mentioning that modeling technology as an exogenous determinant of operating cost may not always be appropriate. To the extent that it reflects a choice by hospital management, the presence of an inferior technology amounts to an inefficiency.

5 In principle, one could also allow for individual cost functions in the RPFM [38]. But for the time being, payment negotiations in Switzerland do not involve hospitals individually but rather by category. Therefore, category-specific estimates are presented in this paper.
}

where $\bar{\alpha} \sim N\left[0, \sigma_{\bar{\alpha}}^{2}\right]$ and $\bar{\beta} \sim N\left[0, \sigma_{\bar{\beta}}^{2}\right]$ have zero mean and variance $\left(\sigma_{u}^{2}, \sigma_{v}^{2}\right)$, respectively. This hierarchical model first measures the mean effects $(\bar{\alpha}, \bar{\beta})$ and then estimates individual effects $\left(\alpha_{j}, \beta_{j}\right)$ for each parameter. The covariance matrix $\Sigma$ is assumed to be Wishart distributed with a $[(K+$ $1) \times(K+1)]$ positive definite covariance matrix $S=\left(\sigma_{\alpha}^{2}, \sigma_{\beta}^{2}, \sigma_{\alpha, \beta}\right)$, denoting unobserved heterogeneity among hospitals. For $\Sigma=0$, no variation exists and the RPFM simplifies to the SFM.

Based on the distributional assumptions made in the SFM and RPFM, Bayesian econometrics is applied for the simultaneous estimation of the parameters contained in the cost frontier and the inefficiency term. Contrary to conventional maximum likelihood inference, unknown parameters are viewed as random variables here, characterized by their prior distributions. The advantage of Bayesian estimation is that it yields exact small sample results. Estimation is performed using R and WinBUGS; Bayesian specifications and programming codes are provided in the "Appendix".

\section{Data and econometric specifications}

\section{Sample}

Swiss hospital data for the time period 2004-2009 come from the annual reports of the Federal Office of Public Health and the Conference of Cantonal Health Ministers. In 2009 they include 333 hospitals, comprising 5 university hospitals (K111), 23 central hospitals (K112), 27 large regional hospitals (K121), 46 medium sized regional hospitals (K122), 46 small regional hospitals (K123), 28 specialized surgery hospitals (K231), and 158 sundry hospitals, viz. psychiatric (K212) and rehabilitation clinics (K221). A total of 127 of the 333 hospitals are private and not subsidized. This is an unbalanced panel because not all hospitals provide information for all the years and a few hospitals have been merged or closed during the observation period.

The entire data set was reviewed and checked for missing data and outliers that could distort the results. Furthermore, all sundry hospitals (e.g., K212 and K221) and non-subsidized hospitals were discarded. After purging the data, an unbalanced panel of about 90 hospitals (amounting to 545 observations from 2004 to 2009) covering the six categories was available. This database includes the following $(\mathrm{CHF}=$ Swiss franc, $1 \mathrm{CHF}=$ 0.8 USD at 2004 exchange rates) variables:

$V C$ : Variable operational expense, in thousands of $\mathrm{CHF}$ $(V C)$

$Y_{1} \quad$ : No. of inpatient cases, CMI-adjusted (CASES)

$Y_{2} \quad$ : Revenue from outpatient treatment, in thousands of CHF (OUTP) 
Table 1 Descriptive statistics of the variables used in the analysis
In $1,000 \mathrm{CHF}, 1 \mathrm{CHF}=0.8$ USD (2004 exchange rates)

In percent; PPS $=78$ in column 3 means that on average $78 \%$ of all hospitals have PPS

\begin{tabular}{lrrrrrrrrr}
\hline Variable & \multicolumn{1}{c}{ Mean } & \multicolumn{1}{c}{ Min } & \multicolumn{1}{l}{ Max } & K111 & K112 & K121 & K122 & K123 & K231 \\
\hline$V C$ & 135,621 & 8,550 & $1,015,756$ & 827,840 & 209,550 & 90,969 & 48,045 & 22,687 & 81,823 \\
$Y_{1}$ & 9,113 & 502 & 52,143 & 43,579 & 15,300 & 6,971 & 3,861 & 1,769 & 5,783 \\
$Y_{2}$ & 27,418 & 0 & 223,937 & 152,727 & 49,468 & 16,679 & 7,844 & 2,953 & 17,904 \\
$P L$ & 101 & 34 & 146 & 108 & 103 & 102 & 99 & 98 & 112 \\
$P M$ & 4 & 2 & 7 & 5 & 4 & 4 & 4 & 4 & 5 \\
$K$ & 229 & 31 & 1,169 & 885 & 396 & 200 & 107 & 63 & 132 \\
$S_{1}$ & 22 & 0 & 134 & 119 & 34 & 15 & 10 & 5 & 8 \\
$S_{2}$ & 39 & 4 & 106 & 66 & 50 & 36 & 33 & 26 & 20 \\
$Z_{1}$ & 78 & 0 & 100 & 50 & 77 & 78 & 86 & 75 & 70 \\
$Z_{2}$ & 14 & 0 & 100 & 10 & 19 & 14 & 15 & 9 & 0 \\
$Z_{3}$ & 64 & 0 & 100 & 40 & 58 & 64 & 71 & 65 & 70 \\
$Z_{4}$ & 51 & 0 & 100 & 50 & 44 & 60 & 54 & 45 & 70 \\
\hline
\end{tabular}

$P L \quad$ : Labor input price, in thousands of CHF $(P L)$

$P M \quad$ : Price of other production inputs, in thousands of CHF $(P M)$

$K \quad$ : No. of beds $(B E D S)$

$S_{1} \quad$ : No. of internship categories $($ INTERN)

$S_{2} \quad$ : No. of specialties (SPEC)

$Z_{1} \quad$ : Dummy $=1$ for all types of prospective payment $(P P S)$

$Z_{2} \quad:$ Dummy $=1$ for payment per patient case (PAYCASE)

$Z_{3} \quad:$ Dummy $=1$ for global budget $(G L O B)$

$Z_{4} \quad:$ Dummy $=1$ for DRG payment $(D R G)$

$T_{t} \quad$ : Year dummies, $t=2005$ to 2009 (base year is 2004)

Summary statistics are shown in Table 1 for the six hospital categories. They suggest that technological heterogeneity between Swiss hospital categories may well have an influence on cost. For example, university hospitals (K111) have the highest variable costs $(V C=827,840)$. This can be attributed in part to their two main outputs $(P C A S E S=43,579$ and $O U T P=152,727)$ and possibly to their comparatively small share of patients with supplementary insurance (18\%, not shown in Table 1$)$. However, they also have the greatest number of internship programs $\left(S_{2}=119\right)$ and specialties $(S P E C=66)$. Specialized hospitals (K231) on the other hand are on average small, with fewer internship programs $(I N T E R N=8)$ and specialties $(S P E C=20)$ while having a high share of supplementary insured patients (47\%, not shown).

Specification of cost frontier

A variable Cobb-Douglas frontier in terms of variable cost (subscripts $i=1, \ldots, N$ and $t=1, \ldots, T$ are dropped for simplicity) can be specified as

$$
\begin{aligned}
\ln \frac{V C}{P M}= & \alpha+\sum_{m=1}^{2} \beta_{m} \ln Y_{m}+\beta_{3} \ln \frac{P L}{P M}+\beta_{4} \ln K \\
& +\sum_{l=1}^{2} \beta_{l} S_{l}+\sum_{t=1}^{5} \beta_{t} T_{t}+u+v
\end{aligned}
$$

Variable cost $(V C)$ depends on two types of output $(Y)$, the input price of labor $(P L)$, the price of other production inputs $(P M)$, capital stock $(K)$, two structural variables $(S)$, and five time dummies $(T)$ to control for any unobserved dynamics over time (base year 2004). Normalizing $V C$ and $P L$ by $P M$ imposes linear homogeneity in input prices.

Health care output - change in patient's health statusand quality of care are difficult to measure in the case of Swiss hospitals. Measures for inpatient care CASES and outpatient care OUTP serve as indicators of intermediate outputs. To adjust for severity in inpatient care, CMIadjusted admissions are used for CASES. Outpatient care is approximated by OUTP revenues from ambulatory care (cf. [3, 13]). This variable is an output indicator because fees are fixed by TARMED, the nationwide fee schedule for ambulatory care services (for a description and critique, see [39]). Unfortunately, indicators of quality such as mortality rates or clinical infection rates are not available because of lacking data. Input price $P L$ is calculated as labor expense divided by the number of full-time employees. Input price $P M$ aggregates over all remaining inputs, such as energy, materiel, and purchased services, again as a result of data limitations. It is calculated as residual cost divided by the number of admissions (for a discussion of this commonly used measure, see p.141 in [10]). Since capital stock (total fixed assets) is not reported by Swiss hospitals, BEDS serves as an approximation. Finally, the number of internship categories INTERN and specialties SPEC control for observable service heterogeneity among hospitals. 
Equation (5) can be justified on several grounds. First, it is compatible with short-term cost minimization, reflecting the fact that capital (indicated by $B E D S$ ) is a predetermined quantity rather than a decision variable. In Switzerland, cantonal hospital planning divisions mainly decide capacity. Second, excluding user cost of capital from the equation avoids measurement error because values would have to be imputed (most hospitals are not charged capital user costs).

\section{Determinants of inefficiency}

Since the influence of PPS on inefficiency is the focus of this article, the inefficiency term is related to additional explanatory variables [see Eq. (2)] in an attempt to test for the two hypotheses stated at the end of Sect. 2:

1. Hospitals under PPS are more cost-efficient than hospitals under RPS.

2. Hospitals under PPS combined with DRG payment are more cost-efficient than those paid with a per diem element.

Hypothesis 1 is tested using three models. Model 1 reads

$\bar{u}_{i t}=\gamma_{o}+\gamma_{1} P P S+\gamma_{2} P^{*} S^{*} D R G+\varsigma_{i t}$.

It relates mean inefficiency to a dummy variable that equals one for hospitals with PPS and zero for hospitals with RPS. The sign of $\gamma_{1}$ determines whether PPS is more cost-effective than the retrospective alternative, the sign of $\gamma_{2}$ pertaining to the interaction term $P P S^{*} D R G$ shows whether in addition to PPS, payment based on DRGs decreases inefficiency even more.

Model 2 is specified as

$$
\begin{aligned}
\bar{u}_{i t}= & \gamma_{o}+\gamma_{1} \text { PAYCASE }+\gamma_{2} G L O B+\gamma_{3} \text { PAYCASE* DRG } \\
& +\gamma_{4} G L O B^{*} D R G+\varsigma_{i t} .
\end{aligned}
$$

This constitutes a refinement of model 1 designed to check for effects of a payment per case and a global budget, respectively. Therefore, the variable PPS is replaced by two dummy variables, GLOB (hospitals on global budget) and PAYCASE (hospitals on payment per case). Hospitals with a retrospective per diem system form the control group. Hospitals paid per patient case are expected to be more cost-efficient (have lower inefficiency scores) than hospitals on a global budget, most of whom enjoy a deficit guarantee or leeway to adjust their budget if confronted with unexpected cost increases (see Sect. 2).

Hypothesis 2 calls for the introduction of an additional dummy variable, $D R G$, in Eqs. (6) and (7). Specified as a nested interaction term, $D R G$ measures the extra effect of DRG payment relative to PPS with a per diem element. As argued in Sect. 2, per diem payment can weaken incentives for cost minimization because hospitals have an incentive to increase length of stay. Therefore, a per diem component in PPS is predicted to result in somewhat higher inefficiency. Conversely, 'pure' DRG payment is predicted to go along with lower inefficiency scores than the frequently used alternative of augmenting PPS with a per diem component.

Hypothesis 2 can be subjected to an additional test using model 3,

$$
\begin{aligned}
\bar{u}_{i t}= & \gamma_{o}+\gamma_{1} P P S+\gamma_{2} P P S^{*} D R G+\gamma_{3} P P S^{*} D R G_{1} \\
& +\gamma_{4} P P S^{*} D R G_{2}+\varsigma_{i t} .
\end{aligned}
$$

This is a refinement of model 1, allowing for a possible catch-up effect of DRG payment over time. It contains two additional dummies $D R G_{\tau}, \tau=1,2$, where $\tau$ indicates the number of years since implementation of DRG payment, reflecting the possibility of DRG payment becoming fully effective with a lag only.

\section{Empirical results}

This section first presents estimates of technology parameters and inefficiency scores (Sect. 5.1), with a special focus on the influence of unobserved heterogeneity. Second, in Sect. 5.2, the influence of PPS is discussed for the three models specified in Sect. 4.3.

To obtain posterior estimates, Monte Carlo Markov Chain (MCMC) algorithms were run for 20,000 iterations, with the first 10,000 samples discarded as a burn-in phase. Different assumptions for priors and starting values converged to roughly the same values without strong periodicities or tendencies in the trace plot. Furthermore, all cost frontier parameters and inefficiency scores have a Monte Carlo error lower than $7.02 * 10^{-4}$, indicating that estimates are quite precise and have reached the equilibrium distribution.

Cost frontier estimates and associated inefficiency scores

Table 2 shows the estimates for the variable cost frontier as specified in Eq. (5). Note that an analysis of cost drivers together with tests for endogeneity, heteroscedasticity, and the skewness of the composite error term had been performed. Hospital output could be endogenous in RPS, which creates incentives for hospitals to increase CASES in a quest for higher revenues. However, a Hausman test did not suggest rejection of the exogeneity assumption. This is also true for the measure of outpatient output (OUTP). Because prices for ambulatory care services provided by 
Table 2 Econometric results for the SFM and RPFM, years 2004 to 2009

a Variable cost $(V C)$ is the dependent variable.

Determinants of inefficiency are shown separately in Table 3

${ }^{\mathrm{b}} \mathrm{SD}$ estimates reveal the diagonal of the covariance matrix $\Sigma$ of Eq. (4)

\begin{tabular}{|c|c|c|c|c|c|c|c|}
\hline \multirow[t]{2}{*}{ Variables $^{\mathrm{a}}$} & \multicolumn{3}{|l|}{ SFM } & \multicolumn{4}{|l|}{ RPFM } \\
\hline & Mean & $2.50(\%)$ & $97.50(\%)$ & Mean & $2.50(\%)$ & $97.50(\%)$ & $\mathrm{SD}^{\mathrm{b}}$ \\
\hline Constant & -0.088 & -0.103 & -0.073 & -0.123 & -0.201 & -0.059 & 0.074 \\
\hline CASES & 0.744 & 0.708 & 0.781 & 0.592 & 0.359 & 0.768 & 0.224 \\
\hline OUTP & 0.004 & -0.002 & 0.011 & 0.076 & -0.045 & 0.217 & 0.158 \\
\hline$P L$ & 0.382 & 0.347 & 0.418 & 0.434 & 0.286 & 0.612 & 0.169 \\
\hline$B E D S$ & 0.220 & 0.182 & 0.258 & 0.281 & 0.118 & 0.482 & 0.159 \\
\hline INTERN & 0.002 & 0.001 & 0.002 & 0.000 & -0.001 & 0.002 & 0.001 \\
\hline$S P E C$ & 0.001 & 0.000 & 0.002 & 0.001 & 0.000 & 0.002 & 0.001 \\
\hline$T_{09}$ & 0.036 & 0.008 & 0.065 & 0.017 & -0.014 & 0.045 & 0.019 \\
\hline$T_{08}$ & 0.023 & -0.005 & 0.051 & 0.007 & -0.013 & 0.031 & 0.009 \\
\hline$T_{07}$ & 0.012 & -0.016 & 0.040 & 0.002 & -0.016 & 0.024 & 0.008 \\
\hline$T_{06}$ & 0.023 & -0.005 & 0.051 & 0.009 & -0.011 & 0.032 & 0.009 \\
\hline$T_{05}$ & 0.009 & -0.017 & 0.034 & 0.004 & -0.015 & 0.028 & 0.011 \\
\hline$\sigma_{u}$ & 0.013 & 0.009 & 0.017 & 0.001 & 0.000 & 0.003 & \\
\hline$\sigma_{v}$ & 0.004 & 0.003 & 0.006 & 0.003 & 0.002 & 0.004 & \\
\hline$D I C$ & $-1,106$ & & & $-1,596$ & & & \\
\hline Obs. & 545 & & & 545 & & & \\
\hline
\end{tabular}

hospitals are determined by TARMED, a nationwide fee schedule, OUTP can safely be considered exogenous. Heteroscedasticity is not a problem either according to a Breusch-Pagan test, except for a weak effect of INTERN on the variance of the composite error term. Finally, because inefficient hospitals by definition lie above the cost frontier, the composite error term needs to be positively skewed. Otherwise, no systematic inefficiency would exist, making OLS sufficient for estimating the cost frontier. However, residuals of the cost driver analysis are positively skewed, indicating that systematic inefficiency exists in the Swiss hospital sector.

The first three columns of Table 2 exhibit the SFM, with its (fixed) parameter estimates and their 2.5 and 97.5 percentile values. Technology parameters satisfy economic restrictions in that variable cost monotonically increases in the outputs CASES and OUTP as well as in the input price $P L$. The only exception is hospital beds $(B E D S)$ with a coefficient of 0.22 , which should be negative because $B E D S$ is a proxy for capital stock [20]. However, BEDS, being correlated with hospital output, might fail to reflect any costreducing effect of substitution of other inputs (in particular, labor) by capital (see, e.g., [14] for similar difficulties). Moreover, variable costs tend to shift up systematically over time, attaining a maximum in $2009\left(T_{09}\right.$, coeff. $\left.=0.036\right)$. Finally, with regard to heterogeneity, it is not surprising to see that more internship categories (INTERN, coeff. $=0.002)$ and a higher number of specialties (SPEC, coeff. $=0.001$ ) have a cost-increasing effect.

On the right hand side of Table 2, estimates for the RPFM are displayed. They differ only slightly from their SFM counterparts [estimation means correspond to the $\bar{\beta}$ of Eq. (4)]. However, entries in the last column suggest a fair amount of variation in the frontier model parameters. They represent the diagonal elements of the covariance matrix $\Sigma$ of Eq. (4), to be interpreted as the variation in the parameters across hospital categories. Heterogeneity is highest for inpatient care (CASES, $\mathrm{SD}=0.224)$, followed by the input price for labor $(P L$, $\mathrm{SD}=0.169)$, capital stock $(B E D S, \mathrm{SD}=0.159)$, and outpatient care $(O U T P, \mathrm{SD}=0.158)$. Note that even though heterogeneity in inpatient care is already adjusted for by a casemix index, a great deal of variation among hospital categories remains. This raises doubts about the relevance of DRG classification as a tool for neutralizing cost variability in inpatient care. However, these conclusions are conditional on the RPFM being superior to the SFM. Therefore, the two models are assessed using the DIC information criterion shown in Table 2 [34]. The lower the DIC value, the better the goodness of fit of the estimated cost frontier, indicating that the RPFM $(D I C=$ $-1596)$ indeed dominates the SFM $(D I C=-1106)$. At least in the case of Swiss hospitals, flexible variants of cost functions are needed to capture all the existing heterogeneity between hospital categories.

An issue of particular importance is the impact of unobserved heterogeneity on estimated inefficiency scores. As shown by Greene [15] and Widmer et al. [38], unmeasured heterogeneity can masquerade as inefficiency $u_{i t}$. The scatter and density plots of Figs. 2 and 3 suggest this to be the case in the present sample. Figure 2 a shows the inefficiency scores of the SFM and the RPFM, which differ strongly between the two models. According to the SFM, the mean inefficiency score is 0.066 , implying that 
Fig. 2 Estimated inefficiency scores of the SFM and RPFM, years 2004-2009. a Density of the SFM and RPFM inefficiency scores. b SFM and RPFM inefficiency scores compared
Fig. 3 Estimated inefficiency scores by model type, years 2004-2009. Density of the a SFM inefficiency scores and b RPFM inefficiency scores
(A) Density of the SFM \& RPFM Inefficiency Scores

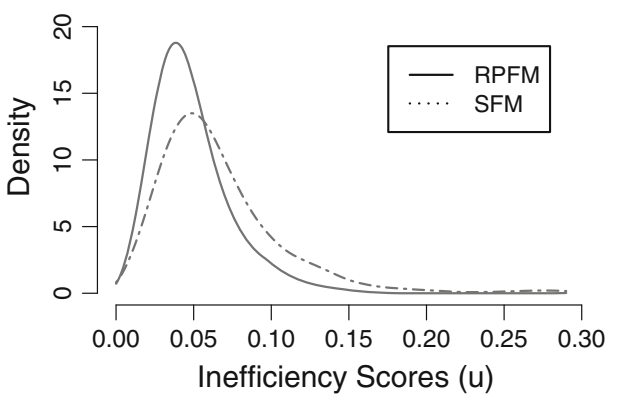

(B) SFM \& RPFM Inefficiency Scores compared

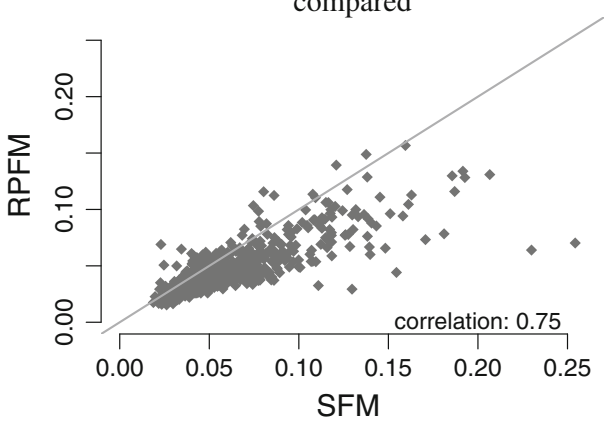

\begin{tabular}{lcccccc}
\hline & Mean & Minimum & Maximum & Std. Dev. & Skewness & Kurtosis \\
\hline SFM & 0.066 & 0.019 & 0.294 & 0.039 & 2.277 & 10.851 \\
RPFM & 0.049 & 0.015 & 0.157 & 0.024 & 1.417 & 5.364 \\
\hline
\end{tabular}

(A) Density of the SFM Inefficiency Scores

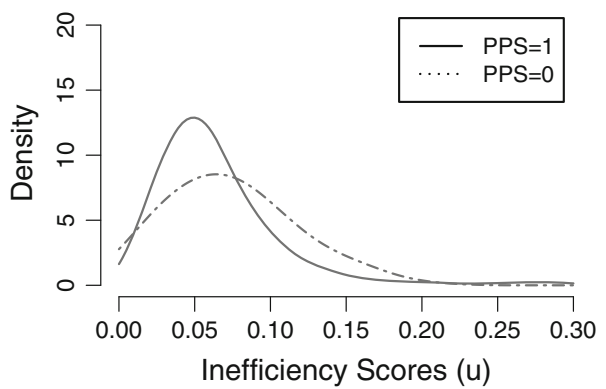

\begin{tabular}{ccccc}
\hline SFM & Mean & Minimum & Maximum & Std. Dev. \\
\hline PPS $=0$ & 0.073 & 0.022 & 0.181 & 0.036 \\
PPS $=1$ & 0.064 & 0.019 & 0.294 & 0.040
\end{tabular}

(B) Density of the RPFM Inefficiency Scores

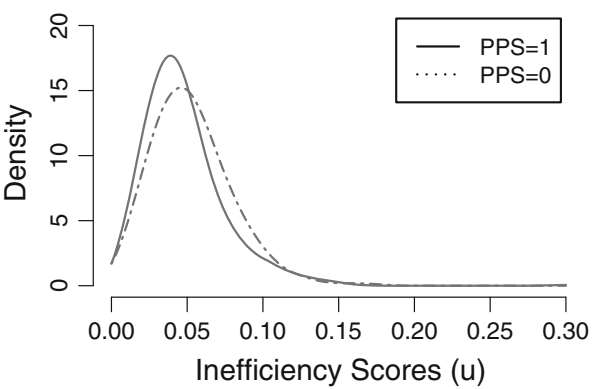

\begin{tabular}{ccccc}
\hline RPFM & Mean & Minimum & Maximum & Std. Dev. \\
\hline PPS $=0$ & 0.053 & 0.017 & 0.157 & 0.024 \\
PPS $=1$ & 0.047 & 0.015 & 0.149 & 0.023 \\
\hline
\end{tabular}

Swiss hospitals could on average reduce their variable cost by $7 \%$. However, according to the RPFM, mean inefficiency falls to about $5 \%$, with 2 percentage points of the SFM scores being attributable to unobserved heterogeneity.

A comparison of the individual scores in Fig. $2 b$ is even more revealing. Although the two models have a high correlation of 0.75 , hospitals appear systematically more inefficient on the basis of the SFM. In particular, hospitals that are rated highly inefficient according to the SFM gain ground when the RPFM is applied. The maximum inefficiency score decreases from 0.294 (SFM) to 0.157 (RPFM), putting the maximum cost reduction at about 16 rather than $29 \%$. At a given point in time and for the majority of Swiss hospitals, it therefore clearly matters whether or not unobserved heterogeneity is taken into account.

It is noteworthy that even the SFM reveals a significantly smaller potential for cost reduction than suggested by previous studies of the Swiss hospital sector (such as $[12,35,38])$. However, since only a subsample of publicly financed hospitals is used here, a detailed comparison is not possible.

Figure 3 reveals some preliminary indications concerning the effectiveness of PPS. Surprisingly, even though SFM scores are known to be biased, both models suggest that PPS reduces hospital cost inefficiency. The main reason is the high correlation of SFM and RPFM inefficiency (see Fig. 2a). According to the SFM, implementation of PPS is associated with a decrease in inefficiency from 0.073 to 0.064 (Fig. 3a) and from 0.053 to 0.047 according to the RPFM (Fig. 3b). Both reductions are significant according to a Wilcoxon rank-sum test (the hypothesis of equal mean inefficiency can be rejected at the $95 \%$ confidence level). However, the estimated decrease based on the SFM (mean = $-0.009)$ is larger than that based on the RPFM (mean $=-0.006)$. Figure 3 also suggests that PPS has an influence on the variance. Under PPS, the distribution of efficiency scores is more concentrated in both panels of Fig. 3. Yet, PPS serves to lower the standard deviation only according to the RPFM (from 0.024 to 0.023 ); according to 
Table 3 Determinants of inefficiency by model type, years 2004-2009

${ }^{\text {a }}$ Mean inefficiency $\bar{u}_{i t}$ is the dependent variable. Technology parameters are not shown; those pertaining to model 1

correspond to those shown in Table 2

\begin{tabular}{|c|c|c|c|c|c|c|}
\hline \multirow[t]{2}{*}{ Variables $^{\mathrm{a}}$} & \multicolumn{3}{|l|}{ SFM } & \multicolumn{3}{|l|}{ RPFM } \\
\hline & Mean & $2.5 \%$ & $97.5 \%$ & Mean & $2.5 \%$ & $97.5 \%$ \\
\hline \multicolumn{7}{|l|}{ Model 1} \\
\hline Constant & -0.13 & -0.31 & 0.01 & -0.17 & -0.35 & -0.04 \\
\hline PPS & 0.02 & -0.09 & 0.12 & -0.01 & -0.15 & 0.09 \\
\hline PPS:DRG & -0.15 & -0.31 & -0.04 & -0.08 & -0.22 & 0.02 \\
\hline \multicolumn{7}{|l|}{ Model 2} \\
\hline Constant & -0.15 & -0.35 & -0.01 & -0.17 & -0.33 & -0.05 \\
\hline PAYCASE & -0.08 & -0.30 & 0.13 & -0.07 & -0.26 & 0.11 \\
\hline GLOB & 0.03 & -0.07 & 0.14 & 0.00 & -0.13 & 0.10 \\
\hline PAYCASE:DRG & -0.06 & -0.29 & 0.14 & -0.04 & -0.24 & 0.15 \\
\hline GLOB:DRG & -0.15 & -0.32 & -0.03 & -0.07 & -0.22 & 0.03 \\
\hline \multicolumn{7}{|l|}{ Model 3} \\
\hline Constant & -0.16 & -0.36 & -0.03 & -0.19 & -0.36 & -0.06 \\
\hline PPS & 0.02 & -0.11 & 0.13 & -0.02 & -0.16 & 0.09 \\
\hline PPS:DRG & -0.13 & -0.33 & 0.03 & -0.08 & -0.25 & 0.07 \\
\hline PPS:DRG1 & -0.08 & -0.29 & 0.10 & -0.03 & -0.21 & 0.14 \\
\hline PPS:DRG2 & -0.01 & -0.25 & 0.18 & -0.02 & -0.19 & 0.15 \\
\hline
\end{tabular}

substantially biases estimates of the influence of $P P S$ on inefficiency. Yet, a $1.7 \%$ decrease in inefficiency remains after controlling for unobserved heterogeneity. Estimates for the interaction term PPS [38] DRG are more intuitive. While SFM and RPFM agree that $D R G$ is negatively correlated with hospital inefficiency, SFM yields on unreasonably large effect (mean coeff $=-0.15$ ). Estimates for $G L O B$ and $G L O B^{*} D R G$ in model 2 again differ significantly between the two approaches. According to the SFM, GLOB has a positive influence (mean coeff. $=0.03$ ) on inefficiency, but not according to the RPFM (mean coeff. $=0.00$ ). Moreover, the SFM interaction term $G L O B^{*} D R G$ indicates an exceedingly high negative effect (mean coeff. $=-0.15$ ) of DRG payment on inefficiency. In model 3, which controls for a possible time lag in the DRG effect, the SFM estimates continue to be systematically larger than the RPFM ones. Also, the SFM estimate of PPS (mean coeff. $=+0.02$ ) again may be biased because of unobserved heterogeneity. Taken together, estimation results differ more strongly between the SFM and RPFM than expected, pointing to a considerable importance of the assumption regarding the production technology of hospitals.

Nevertheless, RPS does appear to undermine cost containment, vindicating hypothesis 1 . At the very least, RPFMbased estimates of models 1 and 3 point to a decrease in inefficiency of about -0.01 thanks to PPS, implying that a switch to PPS causes hospitals to reduce their variable cost by an average of $1 \%$. However, as shown by model 2, efficiency gains depend substantially on whether hospitals are on global budget or on per case payment. While payment per patient case reduces inefficiency by about -0.07 on average, no efficiency gains can be claimed for the global budget (the

\footnotetext{
$\overline{6}$ Technology parameters of models 2 and 3 are not shown. They are found to be comparable to those discussed in Sect. 5.1.
} 
biased SFM estimate is even +0.03 ; it is 0.00 according to the RPFM). Renegotiation that most cantons still allow at the end of the accounting period may well weaken incentives for cost minimization, because this causes hospital managers to anticipate additional financial support.

In Sects. 2 and 4.3, hypothesis 2 was formulated stating that compared to 'pure' DRG payment, incorporating a per diem element serves to increase length of stay and hence variable cost. In Table 3, model 2 allows one to contrast 'pure' DRG classification systems (PAYCASE* DRG or $G L O B^{*} D R G$ reimbursement per patient only) with prospective reimbursement that include a per diem element (reimbursement with a per diem element, GLOB or PAYCASE). Surprisingly, combination with a global budget (mean coeff. $=-0.07$, RPFM estimate) appears to reduce inefficiency even more than the combination with payment per patient case (mean coeff. $=-0.04$ ). However, the full effect of payment per case is larger $(-0.11=-0.07-0.04)$ than that of a global budget $(-0.07=0.00-0.07)$. This makes payment per patient case combined with 'pure' DRG classification the preferable variant for Switzerland from a cost containment point of view. It goes along with $11 \%$ lower inefficiency scores on average compared to RPS. However, model 3 reveals that DRG-based payment is not fully effective during the first year after implementation. Although most cost efficiency gains do occur during the first year $(D R G$, coeff. $=-0.08$ ), the mean coefficient of $D R G_{1}$ and $D R G_{2}$ points to additional gains $(-0.03,-0.02)$ following with a lag of 1 and 2 years, respectively.

These findings are in line with theoretical expectations (see, e.g., $[8,26])$. With respect to the reform of Swiss hospital payment of 2012, they lead to the prediction that PPS will increase average cost efficiency, ceteris paribus, provided implementation is fully prospective and bailouts by cantons are precluded.

\section{Concluding remarks}

The purpose of this article was to estimate the effectiveness of prospective payment in reducing hospital cost inefficiency, using data from Switzerland. The Swiss case is of interest because retrospective per diem payment can be compared with two types of prospective payment, one based on a global budget and another based on payment per patient case. Since the results of previous studies may have been affected by unobserved heterogeneity, two stochastic cost frontier models are specified. The first is the SFM that assumes a homogeneous technology for all hospitals. The second is an RPFM that controls for unobserved heterogeneity through intercepts and slope parameters that are specific to six categories of hospitals. The dependent variable is total operating cost of a varying number of publicly financed Swiss hospitals during the time period of 2004-2009, resulting in 545 observations.

There are two main results from this analysis. First, comparison of the SFM and RPFM reveals a substantial amount of heterogeneity among Swiss hospitals of a given category, causing SFM-based inefficiency scores to be biased upwards by an estimated $2 \%$ on average. The maximum inefficiency score decreases from 0.294 according to the SFM to 0.157 for the RPFM, putting maximum achievable cost savings at approximately $16 \%$. Mean inefficiency decreases from 0.066 (SFM) to 0.049 (RPFM), implying that on average only a cost reduction of $5 \%$ may be achievable. However, unobserved heterogeneity is found to systematically differ between hospital categories, indicating that the SFM is not able to detect the true effect of prospective payment on inefficiency. Therefore, assumptions regarding hospitals' production technology (SFM vs. RPFM) are of crucial importance in the Swiss case.

Second, prospective payment is associated with an increase in hospital cost efficiency. Payment designed to put hospitals at operating risk seems to be effective in reducing hospital cost compared to retrospective payment. However, this benefit may be lost unless cantons firmly preclude bailouts. This statement is based on the finding that payment per case dominates the global budget alternative, which usually provides hospitals with a renegotiation opportunity if they can claim unexpected cost increases to have occurred. In addition, the structuring of payment per case proves important. If combined with DRG classification, it is found to have maximum efficiencyenhancing effect; if combined with a per diem element, incentives for cost minimization are weakened because hospitals may increase length of stay. However, estimates suggest that DRG-based payment is not fully effective right after implementation but induces smaller additional efficiency gains 2-3 years later on. Regarding the reform of hospital payment reform of 2012, these results support the expectations of Swiss policy makers. Per case payment based on DRGs can contain hospital cost, provided that it is fully prospective and precludes bailouts by cantons.

This analysis is not without limitations. First, unobserved heterogeneity is estimated as a time-invariant random variable, causing all time-invariant random noise to be attributed to heterogeneity. Since inefficiency could be time-invariant as well, RPFM estimates underestimate inefficiency. Since the SFM overestimates inefficiency, the true influence of PPS must lie somewhere between the two, making reported results reliable enough to be useful. Second, a more flexible form than the Cobb-Douglas function could have been used for depicting production technology (such as the translog). This choice would permit one to test for specific features 
of technology, in particular homotheticity and economies of scale. Unfortunately, data limitations dictated use of the reduced self-dual Cobb-Douglas form, which by definition imposes constant elasticities of substitution and constant returns to scale. Third, the findings are silent concerning possible effects of PPS on the quality of treatment. Again as a result of lacking data, only a casemix adjustment could be performed. Therefore, the estimated gain in terms of cost efficiency thanks to prospective payment might go along with a decrease in quality. In spite of these limitations, the analysis of this paper not only identifies the effect of prospective payment on hospital inefficiency but also documents the importance of unobserved heterogeneity in correctly measuring inefficiency itself.

\section{Appendix: specifications for Bayesian estimation}

This paper uses Bayesian inference to estimate Eqs. (1) and (3). Inference is made from a posterior distribution $p(\theta \mid X)$ of the unknown parameters (denoted by $\theta$ ) given the observed data (summarized as $X$ ). According to the Bayesian rule, this posterior distribution is given by

$p(\theta \mid X)=\frac{\mathcal{L}(X \mid \theta) p(\theta)}{p(X)} \propto p(\theta) \mathcal{L}(X \mid \theta)$.

It is the product of the prior distribution $p(\theta)$ and the likelihood $\mathcal{L}(X \mid \theta)$, respectively.

For the estimates reported in Sect. 5, the posterior distribution pertaining to the SFM is specified as

$$
\begin{aligned}
& p\left(\alpha, \beta, u, \gamma, \sigma_{v}^{-2}, \sigma_{u}^{-2} ; C, Y, W, Z\right) \\
& \propto p\left(\alpha, \beta, \gamma, \sigma_{v}^{-2}, \sigma_{u}^{-2}\right) \prod_{i=1}^{N} \prod_{t=1}^{T} p\left(u, \gamma, \sigma_{u}^{-2} \mid Z\right) \\
& \times \prod_{i=1}^{N} \prod_{t=1}^{T} \frac{1}{\sqrt{2 \pi \sigma_{v}^{2}}} \exp \left[-\frac{1}{2 \sigma_{v}^{2}}\left(C_{i t}-\left[C\left(Y_{i t}, W_{i t} ; \alpha, \beta\right)+u_{i t}\right]\right)^{2}\right],
\end{aligned}
$$

where $p\left(\alpha, \beta, \gamma, \sigma_{v}^{-2}, \sigma_{u}^{-2}\right)$ are probability distributions of the unknown parameters. The likelihood function in Eq. (10) is as in [16], i.e., normally distributed with $\sigma_{v}^{2}$ which denotes the variance of the random noise $v_{i t}=C_{i t}-\left[C\left(Y_{i t}, W_{i t} ; \alpha, \beta\right)+u_{i t}\right]$. Equation (10) affords gain in flexibility over classical maximum likelihood estimation which requires a joint density function of the random noise and the inefficiency term to be specified. Here, only random noise enters the likelihood function. Inefficiency is estimated hierarchically as a latent variable along with the other parameters of the cost frontier.

Turning to the RPFM, the posterior is given by

$$
\begin{aligned}
& p\left(\alpha, \bar{\alpha}, \beta, \bar{\beta}, u, \gamma, \Sigma, \sigma_{v}^{-2}, \sigma_{u}^{-2} ; C, Y, W, Z\right) \\
& \propto p\left(\bar{\alpha}, \bar{\beta}, \gamma, \Sigma, \sigma_{v}^{-2}, \sigma_{u}^{-2}\right) \prod_{i=1}^{N} \prod_{t=1}^{T} p\left(u, \gamma, \sigma_{u}^{-2} \mid Z\right) \\
& \times \prod_{j=1}^{J}(2 \pi)^{-K / 2}|\Sigma|^{-1 / 2} \\
& \times \exp \left[-\frac{1}{2}\left(\left(\begin{array}{c}
\alpha_{j} \\
\beta_{j}
\end{array}\right)-\left(\begin{array}{c}
\bar{\alpha} \\
\bar{\beta}
\end{array}\right)\right)^{\prime} \Sigma^{-1}\left(\left(\begin{array}{c}
\alpha_{j} \\
\beta_{j}
\end{array}\right)-\left(\begin{array}{c}
\bar{\alpha} \\
\bar{\beta}
\end{array}\right)\right)\right] \\
& \times \prod_{i=1}^{N} \prod_{t=1}^{T} \frac{1}{\sqrt{2 \pi \sigma_{v}^{2}}} \exp \left[-\frac{1}{2 \sigma_{v}^{2}}\left(C_{i t}-\left[C\left(Y_{i t}, W_{i t} ; \alpha_{j}, \beta_{j}\right)+u_{i t}\right]\right)^{2}\right] .
\end{aligned}
$$

Again, the likelihood function is specified as a normal distribution and inefficiency is estimated as a latent variable together with the other unknown parameters. The difference from Eq. (10) lies in the specification of the random intercept $\alpha_{j}$ and the slope parameters $\beta_{j}$, which are estimated at two levels. At the first level, overall determinants of hospital cost $(\bar{\alpha}, \bar{\beta})$ are taken into account, corresponding to the first factor following the proportionally sign of Eq. (11). The second-level estimates concern the effects $\left(\alpha_{j}, \beta_{j}\right)$ associated with six hospital categories as defined in Eq. (4). They are derived from the multivariate normal distribution shown in Eq. (11).

In contrast to classical inference, Bayesian estimation requires information regarding the prior distributions of the unknown parameters, which are considered as random variables. These priors should comprise all information available before the sample data are used. In this paper, the values for the hyperparameters characterizing priors are chosen in a way to imply appropriate priors without imposing excessive restrictions. In particular, the priors for the SFM and RPFM are assumed to be independent,

$$
\begin{aligned}
p\left(\alpha, \beta, \gamma, \sigma_{v}^{-2}, \sigma_{u}^{-2}\right)= & p(\alpha), p(\beta), p(\gamma), p\left(\sigma_{v}^{-2}\right), p\left(\sigma_{u}^{-2}\right) ; \\
p\left(\bar{\alpha}, \bar{\beta}, \gamma, \Sigma, \sigma_{v}^{-2}, \sigma_{u}^{-2}\right)= & p(\bar{\alpha}), p(\bar{\beta}), p(\gamma), \\
& p(\Sigma), p\left(\sigma_{v}^{-2}\right), p\left(\sigma_{u}^{-2}\right) .
\end{aligned}
$$

Here, $p(\alpha)=f_{N}\left[0, \theta_{\alpha}\right], p(\bar{\alpha})=f_{N}\left[0, \theta_{\bar{\alpha}}\right], p(\beta)=f_{N}\left[0, \theta_{\beta}\right]$, $p(\bar{\beta})=f_{N}\left[0, \theta_{\bar{\beta}}\right]$ all have a normal distribution with mean zero and a diffuse prior for their corresponding variance $\theta$. The variance of the likelihood function has a gamma distribution $p\left(\sigma_{v}^{-2}\right)=f_{G}\left[\mu, \theta_{\sigma_{v}^{-2}}\right]$ with diffuse shape and scale parameters. Inefficiency is assumed to be truncated, with normal distribution $p\left(u, \gamma, \sigma_{u}^{2} \mid Z\right)=f_{N}^{+}\left[\gamma Z, \sigma_{u}^{2}\right]$ and $\sigma_{u}^{2}=$ $f_{G}\left[5,\left(5 * \log (\bar{r})^{2}\right)\right] \quad$ and $\quad p(\gamma)=f_{N}\left[0, \theta_{\gamma}\right] /$ $\sqrt{f_{G}\left[5,\left(5 * \log (\bar{r})^{2}\right)\right]}$. This specification is in line with Griffin and Steel [16] and Koop et al. [19], permitting one to 
impose a priori information with regard to mean efficiency, $\overline{e f f}=\exp (-\bar{u})$. Again following Griffin and Steel [16], $\overline{e f f}=0.875$ is assumed for prior efficiency. Finally, the variance of the random parameters is specified as a Wishart distribution $p(\Sigma)=f_{W}[S]$ in accordance with Eq. (11), with diffuse prior for the covariance matrix $S$.

Finally, note that estimates of the unknown parameters can be derived from the marginal posteriors of Eqs. (10) and (11). However, it is not always possible to compute posteriors analytically. Therefore, iterative Monte Carlo Markov Chain (MCMC) simulation is used, which involves iterative sampling from posterior parameter densities. Here, WinBUGS is used to derive these estimates (for an introduction, see [27]). The corresponding computational codes for the SFM and RPFM are shown in Table 4.

Table 4 Computation codes for the standard frontier model and the random parameter frontier model

\begin{tabular}{|c|c|}
\hline Standard Frontier Model & Random Parameter Frontier Model \\
\hline $\begin{array}{l}\text { model }\{ \\
\text { for }(\text { it in } 1: \mathrm{NT})\{ \\
\qquad \text { firm }[\mathrm{it}] \leftarrow \mathrm{n}[\mathrm{it}, 1]\end{array}$ & $\begin{array}{l}\text { model }\{ \\
\text { for( it in } 1: \mathrm{NT})\{ \\
\quad \text { firm }[\mathrm{it}] \leftarrow \mathrm{n}[\mathrm{it}, 1] \\
\quad \operatorname{typ}[\mathrm{it}] \leftarrow \mathrm{n}[\mathrm{it}, 3]\end{array}$ \\
\hline $\begin{array}{l}\text { Likelihood: } \\
\quad \mathrm{Y}[\mathrm{it}] \sim \operatorname{dnorm}(\mathrm{mu}[\mathrm{it}], \mathrm{prec}) \\
\quad \mathrm{mu}[\mathrm{it}] \leftarrow \operatorname{inprod}(\mathrm{b}[1: \mathrm{K}+1], \mathrm{X}[\mathrm{it}, 1: \mathrm{K}+1]) \\
\quad+\mathrm{u}[\mathrm{firm}[\mathrm{it}]] \\
\}\end{array}$ & $\begin{array}{l}\text { Likelihood: } \\
\quad \mathrm{Y}[\mathrm{it}] \sim \operatorname{dnorm}(\operatorname{mu}[\mathrm{it}], \operatorname{prec}) \\
\mathrm{mu}[\mathrm{it}] \leftarrow \operatorname{inprod}(\mathrm{b}[\mathrm{typ}[\mathrm{it}], 1: \mathrm{K}+1], \mathrm{X}[\mathrm{it}, 1: \mathrm{K}+1]) \\
\quad+\mathrm{u}[\text { firm[it] }]\end{array}$ \\
\hline $\begin{array}{l}\text { Hyperpriors: } \\
\text { for (it in } 1: \mathrm{NT})\{ \\
\quad \mathrm{u}[\mathrm{it}] \sim \operatorname{djl} 1 . \mathrm{dnorm} \cdot \operatorname{trunc}(\mathrm{mu} 1[\mathrm{it}], 1 \mathrm{lambda}, 0,1000) \\
\quad \mathrm{mu} 1[\mathrm{it}] \leftarrow \operatorname{inprod}(\mathrm{t}[1: \mathrm{L}+1], \mathrm{Z}[\mathrm{it}, 1: \mathrm{L}+1]) \\
\}\end{array}$ & $\begin{array}{l}\text { Hyperpriors: } \\
\text { for (it in } 1: \mathrm{NT})\{ \\
\quad \mathrm{u}[\mathrm{it}] \sim \mathrm{dj} 1 . \mathrm{dnorm} \cdot \operatorname{trunc}(\mathrm{mu} 1[\mathrm{it}], \mathrm{lambda}, 0,1000) \\
\quad \mathrm{mu} 1[\mathrm{it}] \leftarrow \operatorname{inprod}(\mathrm{t}[1: \mathrm{L}+1], \mathrm{Z}[\mathrm{it}, 1: \mathrm{L}+1]) \\
\} \\
\text { for }(\mathrm{j} \text { in } 1: \mathrm{J})\{ \\
\text { for }(\mathrm{k} \text { in } 1: \mathrm{K}+1)\{ \\
\quad \mathrm{b}[\mathrm{j}, \mathrm{k}] \leftarrow \mathrm{xi} \cdot \mathrm{b}[\mathrm{j}] * \text { b.raw }[\mathrm{j}, \mathrm{k}] \\
\} \quad \text { b.raw }[\mathrm{j}, 1: \mathrm{K}+1] \sim \mathrm{dmnorm}(\mathrm{b} \cdot \text { bar.raw[],b.tau.raw[,]) } \\
\}\end{array}$ \\
\hline $\begin{array}{l}\text { Priors: } \\
\text { for }(\mathrm{k} \text { in } 1: \mathrm{K}+1)\{ \\
\qquad \mathrm{b}[\mathrm{k}] \sim \operatorname{dnorm}(0,0.0001)\end{array}$ & $\begin{array}{l}\text { Priors: } \\
\text { for }(\mathrm{k} \text { in } 1: \mathrm{K}+1)\{ \\
\quad \text { b.bar[k] } \leftarrow \text { xi.b }[\mathrm{k}]^{*} \text { b.bar.raw }[\mathrm{k}] \\
\quad \text { b.bar.raw }[\mathrm{k}] \sim \operatorname{dnorm}(0,0.0001) \\
\quad \text { xi.b }[\mathrm{k}] \sim \operatorname{dunif}(-10,10) \\
\text { \} }\end{array}$ \\
\hline 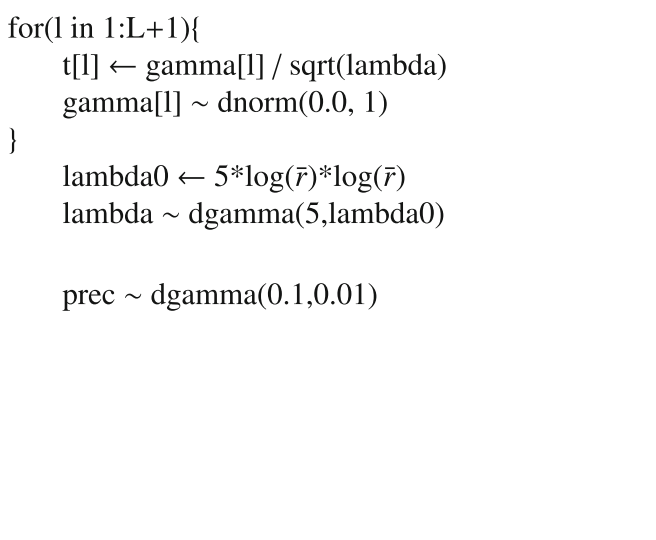 & 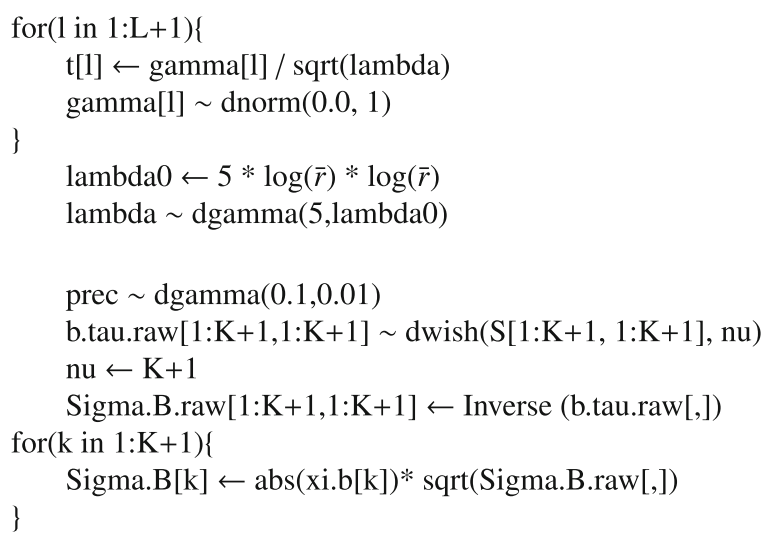 \\
\hline
\end{tabular}




\section{References}

1. Aigner, D., Lovell, C.A.K., Schmidt, P.: Formulation and estimation of stochastic frontier production function models. J. Econ. 6(1), 21-37 (1977)

2. Battese, G.E., Coelli, T.J.: A model for technical inefficiency effects in a stochastic frontier production function for panel data. Empir. Econ. 20(2), 325-332 (1995)

3. Biorn, E., Hagen, T.P., Iversen, T., Magnussen, J.: The effect of activity-based financing on hospital efficiency: A panel data analysis of DEA efficiency scores 1992-2000. Health Care Manag. Sci. 6, 271-283 (2003)

4. Biorn, E., Hagen, T.P., Iversen, T., Magnussen, J.: Heterogeneity in hospitals' responses to a financial reform: a random coefficient analysis of the impact of activity-based financing on efficiency. Working paper 8100, University Library of Munich, Germany (2006)

5. Biorn, E., Hagen, T.P., Iversen, T., Magnussen, J.: How different are hospitals' responses to a financial reform? The impact on efficiency of activity-based financing. Health Care Manag. Sci. 13(1), 1-16 (2010)

6. Borden, J.P.: An assessment of the impact of diagnosis-related group (DRG)-based reimbursement on the technical efficiency of New Jersey hospitals using data envelopment analysis. J. Account. Pub. Policy 7(2), 77-96 (1988)

7. Chalkley, M., Malcomson, J.M.: Contracting for health services when patient demand does not reflect quality. J. Health Econ. 17(1), 1-19 (1998)

8. Chalkley, M., Malcomson, J.M.: Government purchasing for health services. In: Culyer, A.J., Newhouse, J.P. (eds.) Handbook of Health Economics, vol. 1A, pp. 847-889. Elsevier, Amsterdam (2000)

9. Chern, J.-Y., Wan, T.T.H.: The impact of the prospective payment system on the technical efficiency of hospitals. J. Med. Syst. 24, 159-172 (2000)

10. Coelli, T., Rao, D.S.P., O'Donnell, C.J., Battese, G.E.: An Introduction to Efficiency and Productivity Analysis. Springer, Berlin (2005)

11. Dismuke, C., Sena, V.: Has the DRG system influenced the efficiency of diagnostic technology in Portugal? An empirical analysis using parametric and non-parametric methods. Health Care Manag. Sci. 2, 107-116 (1999)

12. Farsi, M., Filippini, M.: An analysis of efficiency and productivity in Swiss hospitals. Swiss J. Econ. Stat. (SJES) 142(I), 1-37 (2006)

13. Farsi, M., Filippini, M., Greene, W.: Application of panel data models in benchmarking analysis of the electricity distribution sector. Ann. Pub. Coop. Econ. 77(3), 271-290 (2006)

14. Filippini, M., Farsi, M., Crivelli, L., Zola, M.: An analysis of efficiency and productivity in Swiss hospitals. Report, Swiss Federal Statistical Office (2004)

15. Greene, W.: Distinguishing between heterogeneity and inefficiency: stochastic frontier analysis of the World Health Organization's panel data on national health care systems. Health Econ. 13(10), 959-980 (2004)

16. Griffin, J., Steel, M.: Bayesian stochastic frontier analysis using WinBUGS. J. Product. Anal. 27, 163-176 (2007)

17. Herwartz, H., Strumann, C.: Hospital efficiency under prospective reimbursement schemes: an empirical assessment for the case of germany. Eur. J. Health Econ. 1-12 (2013). doi:10.1007/ s10198-013-0464-5

18. Huang, C., Liu, J.: Estimation of a non-neutral stochastic frontier production function. J. Product. Anal. 5, 68-80 (1994)

19. Koop, G., Osiewalski, J., Steel, M.F.J.: Bayesian efficiency analysis through individual effects: hospital cost frontiers. J. Econom. 76(1-2), 77-105 (1997)
20. Kumbhakar, S., Lovell, C.A.K.: Stochastic Frontier Analysis: An Econometric Approach. Cambridge University Press, Cambridge (2000)

21. Kumbhakar, S.C., Ghosh, S., McGuckin, J.T.: A generalized production frontier approach for estimating determinants of inefficiency in U.S. dairy farms. J. Bus. Econ. Stat. 9(3), 279-286 (1991)

22. Linna, M.: The impact of health care financing reform on the productivity change in Finnish hospitals. System Analysis Laboratory, Research Reports 79(A) (1999)

23. Meeusen, W., van den Broeck, J.: Efficiency estimation from Cobb-Douglas production functions with composed error. Int. Econ. Rev. 18(2), 435-444 (1977)

24. Meister, U.: Hospitals between regulation and competition (Spitäler zwischen Politik und Wettbewerb: Betriebliche Autonomie im Kantonsvergleich). Report, Avenir Suisse (2008)

25. Morey, R., Dittman, D.: Cost pass-through reimbursement to hospitals and their impacts on operating efficiencies. Ann. Oper. Res. 67, 117-139 (1996)

26. Newhouse, J.P.: Reimbursing health plans and health providers: efficiency in production versus selection. J. Econ. Lit 34(3), 1236-1263 (1996)

27. Ntzoufras, I.: Bayesian Modeling Using WinBUGS. Wiley Series in Computational Statistics. Wiley, Hoboken NJ (2009)

28. Orea, L., Kumbhakar, S.C.: Efficiency measurement using a latent class stochastic frontier model. Empir. Econ. 29(1), 169-183 (2004)

29. O'Reilly, J., Busse, R., Häkkinen, U., Or, Z., Street, A., Wiley, M.: Paying for hospital care: the experience with implementing activity-based funding in five European countries. Health Econ. Policy Law 7(01), 73-101 (2012)

30. Schneider, P.: Provider payment reforms: lessons from Europe and America for South Eastern Europe. Discussion paper, World Bank, Washington (2007)

31. Simar, L., Wilson, P.W.: Statistical inference in nonparametric frontier models: the state of the art. J. Product. Anal. 13, 49-78 (2000)

32. Smith, P.C.: Health care reforms in Europe and their implications for Japan. Jpn. J. Soc. Secur. Policy 3(2), 80-95 (2004)

33. Sommersguter-Reichmann, M.: The impact of the Austrian hospital financing reform on hospital productivity: empirical evidence on efficiency and technology changes using a nonparametric input-based malmquist approach. Health Care Manag. Sci. 3(4), 309-321 (2000)

34. Spiegelhalter, D.J., Best, N.G., Carlin, B.P., van der Linde, A.: Bayesian measures of model complexity and fit. J. R. Stat. Soc. Ser. B 64, 583-640 (2002)

35. Steinmann, L., Zweifel, P.: On the (in)efficiency of Swiss hospitals. Appl. Econom. 35(3), 361-370 (2003)

36. Street, A., O'Reilly, J., Padriac Ward, A.M.: Drg-based hospital payment and efficiency: theory, evidence, and challenges. In: Busse, R., Geissler, A., Quentin, W., Wiley, M. (eds.) DiagnosisRelated Groups in Europe: Moving Towards Transparency, Efficiency and Quality in Hospitals. Open University Press, Maidenhead, pp. 93-114 (2010)

37. Tsionas, E.G.: Stochastic frontier models with random coefficients. J. Appl. Econ. 17(2), 127-147 (2002)

38. Widmer, P.K., Zweifel, P., Farsi, M.: Accounting for heterogeneity in the measurement of hospital performance. Working paper ECON 52, Department of Economics, University of Zürich (2011)

39. Zweifel, P., Tai-Seale, M.: An economic analysis of payment for health care services: the United States and Switzerland compared. Int. J. Health Care Financ. Econ. 9(2), 197-210 (2009) 